\title{
VELOCITY CHARACTERISTICS OF AN HYDRAULIC WEIGHT TRAINING SYSTEM
}

\author{
J. BURNIE, PhD and D. A. BRODIE, PhD
}

School of Physical Education and Recreation, University of Liverpool

\section{INTRODUCTION}

The last twenty years have seen a general increase in health and fitness awareness and with it has come an expansion in the range of strength training equipment now available commercially.

One of the most popular developments has been the multi-station training system which offers a variety of exercise activities incorporated into one large unit. Traditionally, these systems have used conventional weight stacks to provide a fixed resistance throughout a selected range of motion. That is, they offer an isotonic mode of exercise.

As early as 1922 Hill (1922) recognised the limitations of isotonic exercise and suggested that to obtain maximum work from a contracting muscle it would be necessary to oppose its contraction at every stage in the range of motion with a force it was only just able to overcome. This became practicable in the mid 1960's with the introduction of isokinetic exercise, Thistle et al (1966), Hislop and Perrine (1967) and Moffroid et al (1969), and this appears theoretically to fulfil Hill's requirement of a maximum contraction throughout the full range of movement.

In a US patent of 1969 Perrine (1969) legally defines isokinetics. An isokinetic system is unique in two respects. Firstly, the resistance offered is not only variable but totally accommodating, and secondly the active limb functions at a constant pre-selected velocity. The advantages of speed specific exercise are well documented in the literature, Moffroid and Whipple (1970). Maximum transfer gains have been shown to occur when training or rehabilitation is performed at functional velocities.

Safety is a key element inherent in isokinetic systems. When a subject using a constant resistance system experiences pain or fatigue the instinct is to withdraw from the load but unfortunately the weight will continue to load the limb until the subject completes the repetition. This may cause the weight to inflict further damage to the affected joint. Conversely, the reduced force offered by the injured or fatigued subject working on an isokinetic system is immediately matched with an automatically diminished resistance. Thus isotonic systems offer a fixed resistance with subjects working at a variable speed while isokinetic systems offer a totally accommodating resistance at a constant limb velocity.

Multi-station exercise systems utilising hydraulic cams as a means of resistance are commercially available and the promotional literature accompanying these systems often claims that the hydraulically controlled stations function

Address for correspondence:

Dr. J. Burnie

School of Physical Education and Recreation

PO Box 147

Liverpool L69 3BX isokinetically. The purpose of this investigation was to consider the velocity characteristics of the hydraulic system and to assess these with reference to the pre-requisite for a constant angular velocity in an isokinetic system.

The hypotheses tested were:

(a) There should be no significant difference between trials for the subjects at a given setting.

(b) There should be no significant difference between subjects at a given setting.

\section{METHOD}

The system used in this study was an eight station model as specified in Table $\mathrm{I}$.

TABLEI

The eight stations of the hydraulic woight training system

\begin{aligned} & \hline Station Activity \\ & \hline${ }^{* 1}$ Shoulder Press \\ & 2 Dips \\ &$*_{3}$ Bench (Chest) Press \\ &${ }^{*} 4$ High Pulley (Latissimus) \\ &$*_{5}$ Low Pulley \\ & 6 Abdominal Board \\ &${ }^{7}$ Squats \\ & 8 Chins \\ & \hline\end{aligned}

* "Isokinetic" hydraulic stations

The stations said to operate isokinetically are stations 1,3 , 4,5 and 7 , the resistance provided by either single or double oil filled hydraulic cylinders which are adjustable on a scale of 1 to 10 with 0.5 divisions thus altering the position of the fulcrum.

Exeriment 1. Nine healthy subjects ( 5 male, 4 female) gave informed consent to participate in this study. None had previous experience of the system or any other 'isokinetic' devices. Station 7 (squats) of the standard 8 station system was used in this experiment. The resistance is provided by a double hydraulic unit. The shoulder attachments are heavily padded and a heel board suitable for all leg, foot and hip extension exercises was used. Fig. 1 illustrates a subject working at this station.

The side structure of the station was modified to take two magnetically attached photo-electric cells linked to a precalibrated electronic digital timer. A rod $2 \mathrm{~mm}$ in diameter was fixed to the lever arm such that it passed through the beam of the photo-electric cells during the range of motion. The cells were located mid-range such that the angular displacement between the cells was $16^{\circ}$ (see Fig. 2).

The electronic timer was started as the narrow rod passed through the first cell and on passing through the second cell the timer was stopped. In this way the time taken to displace the lever arm through $16^{\circ}$ was recorded. This was reported for six settings on the 1 to 10 scale. 


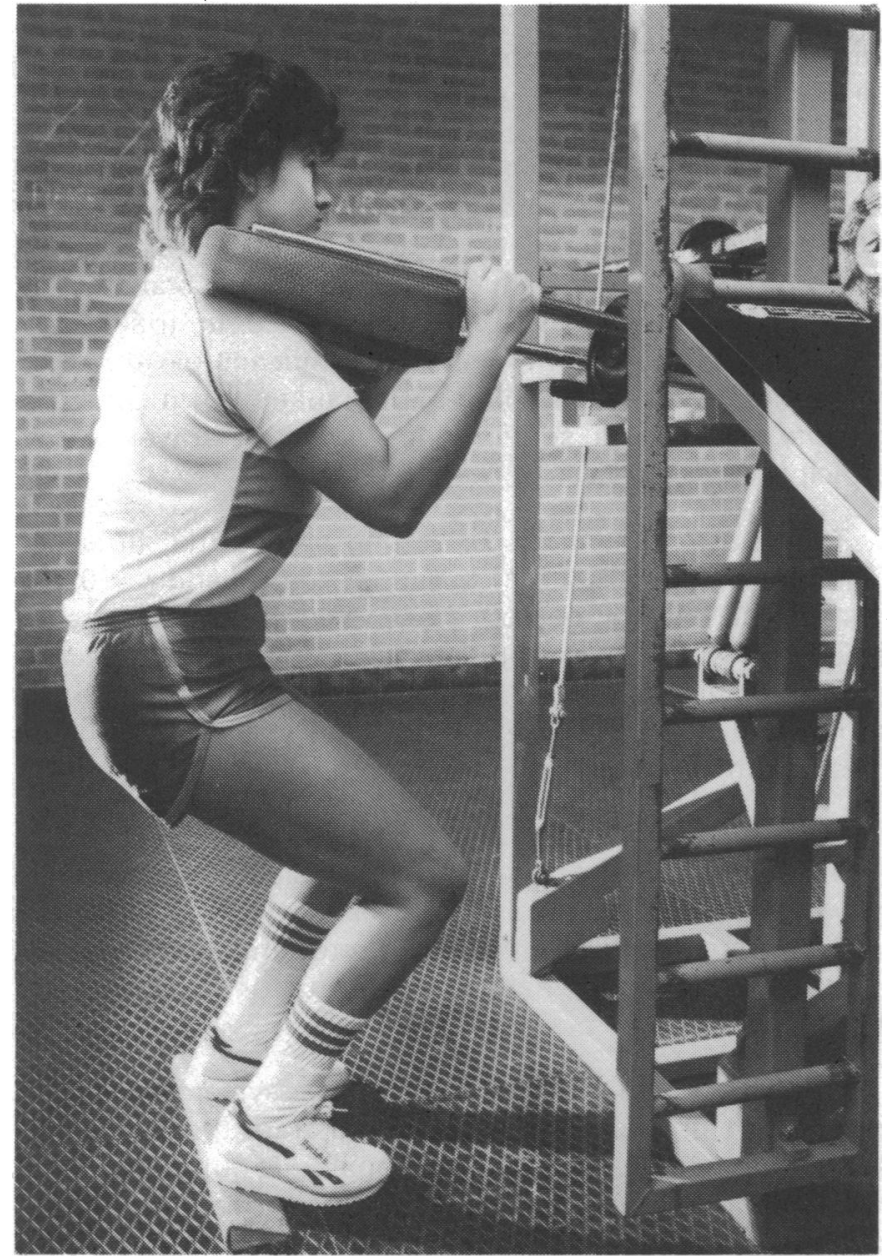

Fig. 1 Subject working at Station 7 - Squats.

Subjects were given a familiarisation period on the squat station, standardised instructions were given and they were then required to perform ten trials at each of the randomised settings, namely $1,6,2,8,3$ and 4 . The time was recorded for each of the ten trials at all six test settings.

Experiment 2. Ten healthy subjects ( 3 female, 7 male) worked on station 1 (shoulder press) in this part of the study. Informed consent, previous experience, photoelectric cell configuration, familiarisation, instructions and setting randomisation were as in Experiment 1.

\section{RESULTS}

Figs. 3 and 4 illustrate the trial times (mean and SD) for all subjects for the squat station $(N=90)$, and the shoulder press station ( $N=100)$, respectively. Table $\|$ gives the

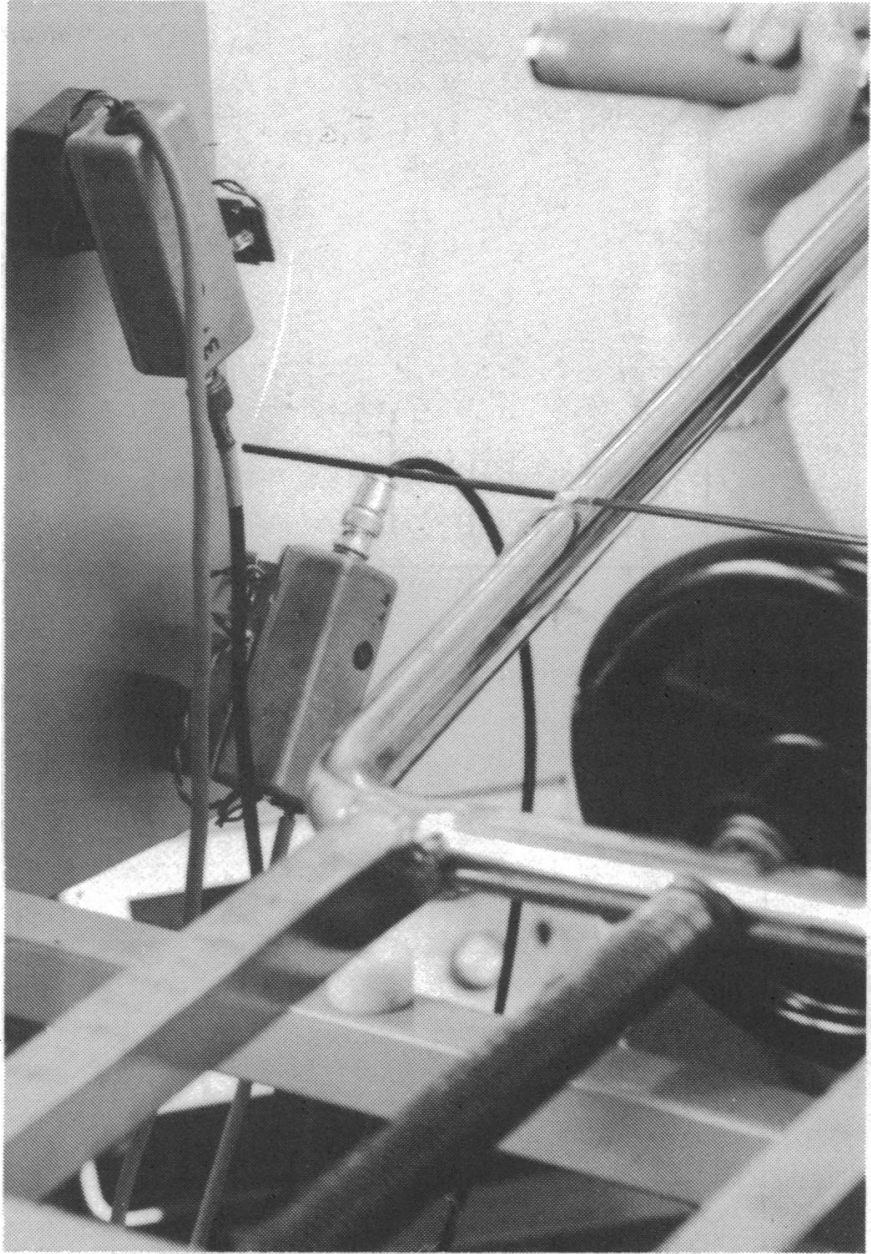

Fig. 2 Modified Station showing the photo-electric cells fitted.

results of a two-way Analysis of Variance (ANOVA) which tests the hypothesis that there is no difference between trials for the subjects at each setting. Results from both Experiments 1 and 2 are given in this table.

Table III gives the results of a two-way ANOVA which tests the hypothesis that there is no significant difference between subjects at each setting. Results from Experiments 1 and 2 are given in this table.

\section{DISCUSSION}

Repeated trials on the squat station show the differences to be non-significant with the exception of the extremes of the range of settings.

At setting 1 the difference in time taken to complete each of the ten trials was shown to be significant at the 0.05 level

TABLE ॥

Results of Two-way Analysis of Variance, 10 trials vs 6 test settings

\begin{tabular}{lcccccccccccc}
\hline Setting & \multicolumn{1}{c}{1} & \multicolumn{2}{c}{2} & & 3 & 4 & & 6 & & 8 \\
\hline Experiment & 1 & 2 & 1 & 2 & 1 & 2 & 1 & 2 & 1 & 2 & 1 & 2 \\
\hline Fvalue & 2.06 & 1.69 & 0.99 & 4.73 & 0.86 & 8.61 & 0.53 & 9.36 & 1.55 & 15.23 & 2.62 & 8.9 \\
$p<$ & 0.05 & N.S. & N.S. & 0.001 & N.S. & 0.001 & N.S. & 0.001 & N.S. & 0.001 & 0.025 & 0.001
\end{tabular}




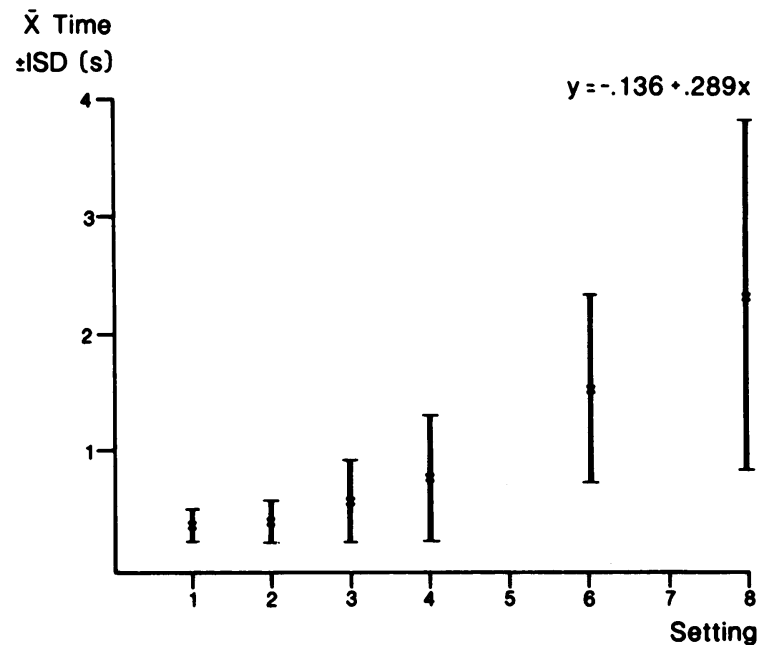

Fig.3 SQUAT

$\bar{x}$ Time

\pm ISD (s)

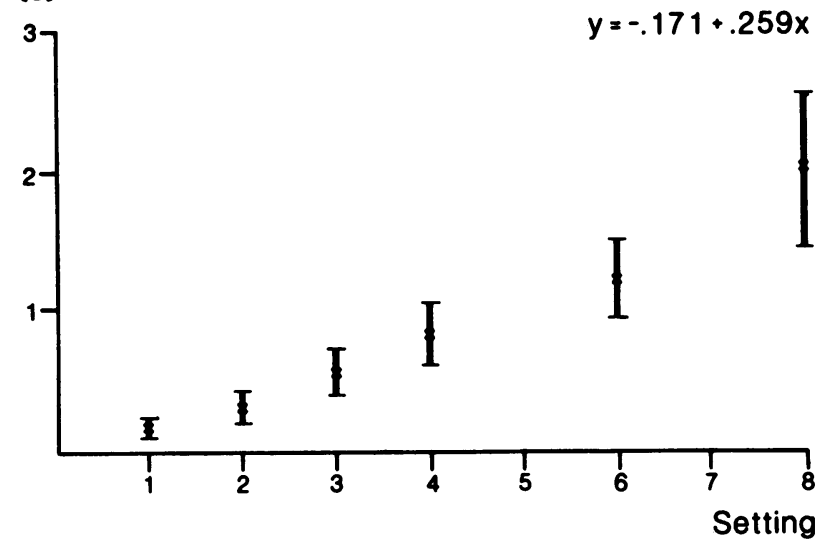

Fig.4 SHOULDER PRESS

while at setting 8 the difference was found to be significant at the 0.025 level.

In the shoulder press the difference in trial times was shown to be significant at the 0.001 level at all settings with the exception of setting 1 where the differences were shown to be non-significant. These results demonstrate that although both stations claim to function on the same principle they do not exhibit the same response characteristics.

The significant difference within intra-subject trial times, on the shoulder press station indicates that this station does not function isokinetically.
In keeping with the isokinetic principle (Perrine, 1969) the time taken by different subjects at a given setting should not be significantly different. A comparison of mean values in the Figs. 3 and 4 illustrate an apparent difference in trial times between subjects at a given setting. For example, in Experiment 1 the mean trial time for subject 9 at setting 8 was $0.52 \mathrm{sec}$ compared to a mean time of $5.27 \mathrm{sec}$ for subject 2 at the same setting. When examined statistically, using a two-way ANOVA, the difference in trial times between subjects was shown to be significant $(p<0.001)$ at all settings - see Table III. This fluctuation in movement velocity at a given setting once again contravenes the principle of isokinetics.

The linear correlations of setting versus trial time are high, $r=0.975$ ( $p<0.001$ ) for squat station and $r=0.992$ ( $p<0.001$ ) for shoulder press. The linear regression equations which best represent the regression of time on setting are $y=-0.136+0.289 x$ and $y=-0.171+0.259 x$ for squat and shoulder press respectively. Figs. 3 and 4 illustrate the relationship.

Although not totally accommodating, this mode of resistance is superior to the fixed resistance offered in conventional isotonic weight stacks. They also exhibit an inherent safety feature with the self-returning mechanism at the hydraulic stations, particularly useful for those working in rehabilitation.

One very useful development of this system would be some form of digital measure to illustrate the maximum force produced or alternatively an analogue output showing the force-time curve for the range of movement. Visual incentive and immediate feedback of this nature are helpful for both the elite athlete following an intensive strength training programme and the patient following a prescribed rehabilitation programme. A hydraulic system of this type which does not provide a value for forces generated is used less frequently by those who wish to quantify changes in performance.

\section{References}

Hill, A. V., 1922 "The maximum work and mechanical efficiency of human muscles and their most economical speed". J.Physiol. 56: 19-41.

Hislop, H. J., Perrine, J. J., 1967 "The isokinetic concept of exercise". Physical Therapy 47: 2, 114-117.

Moffroid, M., Whipple, R., Hofkosh, J., Lowman, E. and Thirtle, H., 1969 "A study of isokinetic exercise". Physical Therapy 49: 7, 735-747.

Moffroid, M. T. and Whipple, R. H., 1970 "Specificity of speed of exercise". Adapted in part from a Thesis written for the degree of MA from New York University, New York.

Perrine, J. J., 1969 "Isokinetic exercise process and apparatus". United States Patent Office No. 3,465,592.

Thistle, H. G., Hislop, H. J., Moffroid, M. and Lowman, E. W., 1966 “Isokinetic contraction: a new concept of resistive exercise". Archives of Physical Medicine and Rehabilitation 48: 279-282.

TABLE III

Results of Two-way Analysis of Variance, subjects (Experiment 1, $n=9$; Experiment 2, $n=10$ ) vs 6 settings

\begin{tabular}{lccccccccccccc}
\hline Setting & \multicolumn{1}{c}{} & \multicolumn{2}{c}{2} & \multicolumn{2}{c}{3} & \multicolumn{2}{c}{4} & \multicolumn{3}{c}{8} & \multicolumn{2}{c}{8} \\
\hline Experiment & 1 & $2^{*}$ & 1 & $2^{*}$ & 1 & $2^{*}$ & 1 & $2^{*}$ & 1 & $2^{*}$ & 1 & $2^{*}$ \\
\hline Fvalue & 35.73 & 41.14 & 163.34 & 254.8 & 16.31 & 186.5 & 17.85 & 200.9 & 189.6 & 235.0 & 334.2 & 103.0 \\
p $<$ & 0.001 & 0.001 & 0.001 & 0.001 & 0.001 & 0.001 & 0.001 & 0.001 & 0.001 & 0.001 & 0.001 & 0.001 \\
\hline
\end{tabular}

